\title{
The Status of Inflammatory Factors Involved in Coronary Artery Disease in Veteran Football Players
}

\author{
Mohsen Jafari (iD) ${ }^{1, *}$ \\ ${ }^{1}$ Department of Sport Sciences, Shirvan Branch, Islamic Azad University, Shirvan, Iran \\ "Corresponding author: Department of Sport Sciences, Shirvan Branch, Islamic Azad University, Shirvan, Iran. Email: sport87mohsen@gmail.com
}

Received 2019 July 28; Revised 2019 October 19; Accepted 2019 October 20.

\begin{abstract}
Background: Homocysteine, C-reactive protein (CRP), fibrinogen, and lipoprotein (a) (LPA) are four novel cardiovascular risk factors.

Objectives: The purpose of this study was to compare the serum levels of these substances in veteran football players and nonathletes.

Methods: The subjects of this study were 16 male orthopedic veterans assigned to the two control and experimental groups. The age range was 40 to 50 years old. The athlete group was players of North Khorasan veterans' team of futsal that trained regularly in the past six months. Trainings of the athlete group were three sessions per week that each session consisted of a warm-up, technical, tactical, and fitness trainings and cooling down. Non-athlete subjects had no regular trainings in the past six months. After 12 hours of fasting, serum levels of dependent variables were evaluated. Independent $t$-test was used for computing the mean differences of dependent variables between the two groups.

Results: The findings showed that mean levels of homocysteine $(\mathrm{P}=0.034)$, fibrinogen $(\mathrm{P}=0.001)$ and LPA $(\mathrm{P}=0.022)$ were lower in the experimental group compared to the control group $(\mathrm{P} \leq 0.05)$. There was no significant difference between the two groups in terms of CRP mean levels $(\mathrm{P}=0.072)$.

Conclusions: Generally, orthopedic veterans can prevent cardiac events through regular futsal training because this type of training may reduce the levels of homocysteine, fibrinogen and LPA.
\end{abstract}

Keywords: Fibrinogen, Homocysteine, CRP, Lipoprotein (a), Football

\section{Background}

Over the past few decades, coronary artery disease (CAD) or atherosclerosis are the main causes of most deaths worldwide (1). Homocysteine, C-reactive protein (CRP), fibrinogen and lipoprotein (a) (LPA) are among cardiovascular risk factors $(2,3)$. Homocysteine is involved in atherosclerosis by stimulating the accumulation of low-density lipoprotein (LDL) and the formation of foam cells and suppressing the proliferation of endothelial cells. Moreover, CRP increases the expression of adhesion molecules, stimulates LDL phagocytosis by macrophages, and suppresses the expression of nitric oxide synthase. Fibrinogen plays a pivotal role in the formation of the blood clot and its viscosity, all of which can increase the risk of myocardial infarction (2).

Increasing LPA raises the risk of atherosclerosis by two mechanisms because, in addition to its cholesterol deposition in the vasculature, it has precocious and antifibrinolytic properties due to its similar structure with plasminogen and plasmin and the lack of fibrinolytic activity (3). Physical activity and regular exercise have beneficial effects on the prevention and treatment of atherosclerosis and it has been proven in numerous studies (3); however, there is not much research on the effect and relationship between physical activity and exercise with homocysteine, CRP, fibrinogen, and LPA in veterans. Kuo et al. (4) investigated the relationship between homocysteine and cardiovascular fitness. The results showed that homocysteine levels had an inverse relationship with cardiovascular fitness in females and not in males. Unt et al. (5) examined the effect of physical activity and physical fitness on homocysteine in elite male athletes. The findings showed that physical activity and aerobic fitness are inversely related to homocysteine levels. In another study, Fedewa et al. (6) stated that regular exercise reduces CRP levels. Gomez-Marcos et al. (7) investigated the relationship between regular physical activity with fibrinogen concentrations in middle-aged males and females and showed that physical activity has an 
inverse relationship on plasma fibrinogen concentrations. Bermudez et al. (8) showed that regular physical activity decreases LPA.

\section{Objectives}

Owing to the lack of research on the relationship between physical activity with homocysteine, CRP, fibrinogen and LPA among male veterans, the purpose of this study was to compare the rates of these variables among male athletic veterans and non-athlete veterans.

\section{Methods}

The statistical population of this study was orthopedic male veterans in Bojnourd county. Sixteen male veterans were selected by using accessible method and were divided into two groups: athlete $(\mathrm{N}=8)$ and non-athlete $(\mathrm{N}=8)$ (Table 1). The subjects were all healthy (no chronic and metabolic diseases) and overweight $(25<\mathrm{BMI}<30)$ and were not chemical veterans, so they did not use any special medication to affect the dependent variables of the study, and their injuries were caused by bullet or cracks in the jaw, leg, and body. Informed consent was obtained from the subjects before the study. This study was confirmed by the Ethics Committee of Islamic Azad University (Bojnourd Branch). The athlete group included the members of the futsal team of veterans who were being prepared for national competitions and were practicing regularly during the last six months. The athlete's training sessions consisted of three sessions a week, each session included five minutes of preparing, 10 minutes to warm-up, 70 minutes of the main training, including technical, tacti$\mathrm{cal}$, and bodybuilding exercises, and 10 minutes of cooling down with slow running and stretching exercises. The athlete group held a friendly match with one of the provincial teams each week, in addition to three training sessions. Non-athlete subjects did not have any regular training during the past six months.

After measuring the height and weight of the subjects, they were advised to have a fasting period of 12 hours and, after this time, they were sent to the medical diagnostic laboratory to determine the levels of dependent variables. The subjects did not undergo any intensive physical activity until two days prior to referral to the lab. Blood samples were taken from the left ventricle of the subjects, and then blood samples were placed in an icebox after centrifugation and sent to the medical diagnostic laboratory in Mashhad. Serum levels of fibrinogen and LPA were measured using an autoanalyzer. Serum levels of homocysteine (ELISA and specialty kit (My BioSource made in Amer- ica)) were measured in $\mathrm{mg} / \mathrm{L}$ and CRP (Immunoturbidometric method and Pars Test kit (Iran)) (mg/dL) using an autoanalyzer unit size.

For statistical calculations, at first the normal data distribution was examined by Shapiro-Wilk test and after determining it, independent $t$-test was used to calculate the difference between the mean of dependent variables between the two groups.

\section{Results}

The distribution of the data was normal between the groups (Table 2). The height, weight, and BMI values did not differ significantly between the two groups $(\mathrm{P}>0.05)$; thus the subjects were homogeneous in terms of physical characteristics. Analysis of the findings showed that homocysteine $(\mathrm{P}=0.034)$, fibrinogen $(\mathrm{P}=0.001)$, and LPA ( $\mathrm{P}$ $=0.022)$ were significantly lower in athletes than in nonathletes $(\mathrm{P} \leq 0.05)$; meanwhile, this difference was not significant in terms of CRP levels $(\mathrm{P}=0.262)$ (Table 3$)$.

\section{Discussion}

In this study, for the first time in Iran, the amount of homocysteine, CRP, fibrinogen, and LPA was investigated in the two orthopedic athletic veteran and non-athletic groups.

Therefore, there are no studies comparing the amount of these materials between athletic and nonathletic men. Serum fibrinogen levels in the athlete group were significantly lower than that of the non-athlete group. The mechanisms that can reduce fibrinogen levels are as follows: increasing plasma volume, developing cardiovascular function, and improving lipid profiles. Fibrinogen levels of blood have also an inverse relationship with stress and obesity, which may have been improved in the subjects of this study. Interleukin-6 is also a hormone secreted from adipose tissue and secretes fibrinogen from this organ by affecting the liver. Therefore, the reduction of the fat percentage in athletes reduces the secretion of interleukin- 6 and subsequently, reduces fibrinogen levels in the blood (2).

Another finding of this study was the lower level of LPA in athletes compared to non-athletes, which was not in line with the findings of Kadoglou et al. (9). Kadoglou et al. (9) showed that resistance training significantly reduced glycemic index, insulin resistance, systolic blood pressure and APOB, but it had no significant effect on APOA1, LPA, fibrinogen, and lipid profiles. Analysis of the findings showed that homocysteine levels were significantly lower in the athlete group than non-athlete group. The findings of this 


\begin{tabular}{|c|c|c|c|c|c|c|}
\hline Group & & & Height, $\mathrm{m}$ & & & BMI, kg.m ${ }^{-2}$ \\
\hline Athletes & & $=2.9$ & $1.7 \pm 0.03$ & & & $26.4 \pm 1.5$ \\
\hline Non-athletes & & 1.06 & $1.7 \pm 0.06$ & & & $28.4 \pm 3.26$ \\
\hline Differences $^{\mathrm{a}}$ (P value) & & & 0.68 & & & 0.12 \\
\hline \multicolumn{7}{|c|}{${ }^{\mathrm{a}}$ Differences are based on independent sample $t$-test. } \\
\hline \multicolumn{7}{|l|}{ Table 2. Tests of Normality } \\
\hline \multirow{2}{*}{ Variables } & \multicolumn{3}{|c|}{ Kolmogorov-Smirnov } & \multicolumn{3}{|c|}{ Shapiro-Wilk } \\
\hline & Statistic & df & PValue & Statistic & df & P Value \\
\hline Fibrinogen & 0.143 & 16 & 0.20 & 0.966 & 16 & 0.777 \\
\hline Homocysteine & 0.106 & 16 & 0.20 & 0.965 & 16 & 0.745 \\
\hline Lipoprotein(a) & 0.129 & 16 & 0.20 & 0.940 & 16 & 0.344 \\
\hline CRP & 0.152 & 16 & 0.20 & 0.966 & 16 & 0.769 \\
\hline
\end{tabular}

$\overline{\text { Table 3. Comparison of Dependent Variables Between the Groups (Independent } T \text { - }}$ Test Results)

\begin{tabular}{|c|c|c|c|}
\hline Variable & Athletes & Non-Athletes & P Value \\
\hline Fibrinogen, mg.dL $^{-1}$ & $255.9 \pm 15.8$ & $287.1 \pm 15.7$ & 0.001 \\
\hline $\mathbf{L P}(\mathbf{a}), \mathrm{mg}^{-\mathbf{L}^{-1}}$ & $123.25 \pm 34.66$ & $162.75 \pm 26$ & 0.022 \\
\hline Homocysteine, mmol.L $\mathrm{L}^{-1}$ & $7.35 \pm 1.2$ & $8.9 \pm 1.3$ & 0.034 \\
\hline hsCRP, mg.dL ${ }^{-1}$ & $1.2 \pm 0.7$ & $1.5 \pm 0.3$ & 0.262 \\
\hline
\end{tabular}

study were similar to those of Unt et al. (5) but it was not in line with the findings of Kuo et al. (4). In a study conducted by Unt et al. (5), it was shown athletes had lower levels of homocysteine than non-athletes. Kuo et al. (4), the homocysteine levels had an inverse relationship with cardiovascular fitness in men.

Another finding of this study was that there was no significant difference in CRP levels between athlete and nonathlete groups. The findings of this study on CRP were similar to those of Rombaldi et al. (10), but it was not in line with findings by Fields et al. (11) and Loprinzi et al. (12). Rombaldi et al. (10) in a cross-sectional study examined the relationship between CRP levels and physical activity in 2,213 men aged 18 to 23 years. The results showed that there is no relationship between CRP levels and physical activity. Fields et al. (11) reported that in injured war men, high physical activity has a relationship with low CRP. Loprinzi et al. (12) studied the relationship between physical activity and CRP in 2,912 adults and 1,643 children examined and reported that there was an inverse relationship between the above factors in adults and children. Regarding the fact that in the present study, CRP levels were compared between athlete and non-athlete groups, and there was a significant decrease in the CRP level in the athlete group $(\mathrm{P}=0.07)$, we cannot precisely compare the results of this study with previous studies. Possibly, if the athlete subjects did more exercises in this study, the difference in CRP between them and the non-athlete group would be significant, while the sampling and laboratory errors could not be neglected. Reducing fat percentage leads to reductions in adipocytokines such as the tumor necrosis factoralpha and its receptors, as well as interleukin-6, and since these agents stimulate the synthesis of CRP in hepatocytes, reducing the fat percentage from regular physical activity can reduce CRP (2). Overall, the findings of this study showed that homocysteine, fibrinogen, and LPA were significantly higher in athlete veterans, and CRP levels were significantly less than non-athlete veterans, indicating a reduction in the risk of atherogenesis. Therefore, it is recommended to use futsal exercises to prevent cardiovascular events.

\section{Footnotes}

Conflict of Interests: There is no conflict of interest to declare.

Ethical Approval: This study was confirmed by the Ethics Committee of Islamic Azad University of Bojnourd.

Funding/Support: There is no funding support.

\section{References}

1. Nesa Lesa K, Ferdous R, Alam F. A comparative studies of nutritional status, physical activity and life style between male and female patients of coronary heart disease in Khulna city, Bangladesh. Am J Health Res. 2015;3(5):270. doi: 10.11648/j.ajhr.20150305.12. 
2. Bizheh N, Jaafari M. The effect of a single bout circuit resistance exercise on homocysteine, hs-CRP and fibrinogen in sedentary middle aged men. Iran J Basic Med Sci. 2011;14(6):568-73. [PubMed: 23493183]. [PubMed Central: PMC3586852].

3. Nordestgaard BG, Chapman MJ, Ray K, Boren J, Andreotti F, Watts GF, et al. Lipoprotein(a) as a cardiovascular risk factor: Current status. Eur Heart J. 2010;31(23):2844-53. doi: 10.1093/eurheartj/ehq386. [PubMed: 20965889]. [PubMed Central: PMC3295201].

4. Kuo HK, Yen CJ, Bean JF. Levels of homocysteine are inversely associated with cardiovascular fitness in women, but not in men: Data from the National Health and Nutrition Examination Survey 1999-2002. J Intern Med. 2005;258(4):328-35. doi: 10.1111/j.1365-2796.2005.01546.x. [PubMed: 16164571].

5. Unt E,Zilmer K, Magi A, KullisaarT, Kairane C, Zilmer M. Homocysteine status in former top-level male athletes: Possible effect of physical activity and physical fitness. Scand JMed Sci Sports. 2008;18(3):360-6. doi: 10.1111/j.1600-0838.2007.00674.x. [PubMed: 17555540].

6. Fedewa MV, Hathaway ED, Ward-Ritacco CL. Effect of exercise training on c reactive protein: A systematic review and meta-analysis of randomised and non-randomised controlled trials. Br J Sports Med. 2017;51(8):670-6. doi: 10.1136/bjsports-2016-095999. [PubMed: 27445361].

7. Gomez-Marcos MA, Recio-Rodriguez JI, Patino-Alonso MC, MartinezVizcaino V, Martin-Borras C, de-la-Cal-Dela-Fuente A, et al. Relationship between physical activity and plasma fibrinogen concentrations in adults without chronic diseases. PLoS One. 2014;9(2). e87954. doi: 10.1371/journal.pone.0087954. [PubMed: 24498413]. [PubMed Central: PMC3912191].

8. Bermudez V, Aparicio D, Rojas E, Penaranda L, Finol F, Acosta L, et al. An elevated level of physical activity is associated with normal lipoprotein(a) levels in individuals from Maracaibo, Venezuela. Am J Ther. 2010;17(3):341-50. doi: 10.1097/MJT.0b013e3181c1236e. [PubMed: 20019590].

9. Kadoglou NP, Fotiadis G, Athanasiadou Z, Vitta I, Lampropoulos S, Vrabas IS. The effects of resistance training on ApoB/ApoA-I ratio, $\mathrm{Lp}(\mathrm{a})$ and inflammatory markers in patients with type 2 diabetes. Endocrine. 2012;42(3):561-9. doi: 10.1007/s12020-012-9650-y. [PubMed: 22407494].

10. Rombaldi AJ, Pellanda LC, Bielemann RM, Gigante DP, Hallal PC, Horta BL. Cross-sectional and prospective associations between physical activity and C-reactive protein in males. PLoS One. 2015;10(5). e0125984. doi:10.1371/journal.pone.0125984. [PubMed: 25961844]. [PubMed Central: PMC4427448].

11. Fields AJ, Hoyt RE, Linnville SE, Moore JL. Physical activity, sleep, and C reactive protein as markers of positive health in resilient older men. J Health Psychol. 2016;21(9):1928-38. doi: 10.1177/1359105314568578. [PubMed: 25673372].

12. Loprinzi P, Cardinal B, Crespo C, Brodowicz G, Andersen R, Sullivan E, et al. Objectively measured physical activity and C-reactive protein: National Health and Nutrition Examination Survey 2003-2004. Scand J Med Sci Sports. 2013;23(2):164-70. doi: 10.1111/j.1600-0838.2011.01356.x. [PubMed: 21812825]. [PubMed Central: PMC3959887]. 